\title{
Publisher's Note: Accurate force field for molybdenum by machine learning large materials data
} [Phys. Rev. Materials 1, 043603 (2017)]

\author{
Chi Chen, Zhi Deng, Richard Tran, Hanmei Tang, Iek-Heng Chu, and Shyue Ping Ong
}

Q (Received 24 October 2018; published 30 October 2018)

DOI: 10.1103/PhysRevMaterials.2.109902

This paper was published online on 15 September 2017 with incorrect images for Figs. 3(c) and 9. The figures have been replaced online as of 23 October 2018. 